\title{
Neutronics Analysis for the ITER Tritium and Deposit Monitor Diagnostics
}

\author{
Arkady Serikov $^{\mathrm{a}^{*}}$, Luciano Bertalot $^{\mathrm{b}}$, Ulrich Fischer ${ }^{\mathrm{a}}$, David Anthoine ${ }^{\mathrm{b}}$, Christophe Penot $^{\mathrm{b}}$ \\ ${ }^{a}$ Karlsruhe Institute of Technology (KIT), Institute for Neutron Physics and Reactor Technology, Hermann- \\ von-Helmholtz-Platz 1, 76344 Eggenstein-Leopoldshafen, Germany \\ ${ }^{b}$ ITER Organization, Route de Vinon-sur-Verdon, CS 90 046, 13067 St. Paul Lez Durance Cedex, France \\ *Corresponding author: arkady.serikov@kit.edu
}

This paper presents new results of neutronics analysis performed in support for the design development of the Tritium and Deposit Monitor (TDM) to be installed inside the ITER Equatorial Port Plug (EPP) \#17. This monitor is a laser based diagnostics to provide information about the tritium content in the deposited layer on the inner baffle of the ITER divertor. Neutronics analysis is performed with the local MCNP models of EPP\#17 which comprises two adjacent diagnostics: TDM and Core-Imaging X-ray Spectrometer (CIXS). The MCNP 3D models were converted from the corresponding CAD models. Critical neutronics issues related to radiation streaming, nuclear heating and activation are discussed and found shielding design solutions are presented in this paper. It was proposed to increase the length of one segment between two particular mirrors in the labyrinth optical mirror system to prevent direct neutron streaming from the plasma to the optical box in Port Interspace (PI), where maintenance is planned $\sim 12$ days after shutdown. Shield block made of boron carbide behind the optical box was added. To provide the possibility of personnel access to the PI area, Shut-Down Dose Rate (SDDR) has been calculated with the results presented as map distributions and estimates in spherical detectors. Using the Rigorous 2-Step mesh-based (R2Smesh) method of SDDR calculations which combines the FISPACT activation and MCNP transport allowed us to distinguish different decay gamma sources in forming the SDDR. The SDDR results are presented for two variants of the EPP17-CIXS models, with and without the monitor. Therefore the contribution to SDDR from the monitor was deduced. This work provides only the relative values of SDDR. The absolute values will be obtained after the finalizing of the designing work for all the EPP17 diagnostics. This is a forthcoming task of EPP17 diagnostics integration into the ITER-global C-lite MCNP model.

Keywords: Fusion neutronics, ITER, Tritium and Deposit Monitor Diagnostics, Equatorial Port \#17, MCNP

\begin{tabular}{l|l}
\hline Topic Category & D. Diagnostics, Data Acquisition and Remote Participation \\
\hline Presentation Preference & Oral Presentation \\
\hline
\end{tabular}

